LAWRENCE LIVERMORE N A TION AL LABORATORY
Real-space formulation of the electrostatic potential and total energy of solids

J. E. Pask, P. A. Sterne

May 13, 2004

Physical Review B 
This document was prepared as an account of work sponsored by an agency of the United States Government. Neither the United States Government nor the University of California nor any of their employees, makes any warranty, express or implied, or assumes any legal liability or responsibility for the accuracy, completeness, or usefulness of any information, apparatus, product, or process disclosed, or represents that its use would not infringe privately owned rights. Reference herein to any specific commercial product, process, or service by trade name, trademark, manufacturer, or otherwise, does not necessarily constitute or imply its endorsement, recommendation, or favoring by the United States Government or the University of California. The views and opinions of authors expressed herein do not necessarily state or reflect those of the United States Government or the University of California, and shall not be used for advertising or product endorsement purposes. 


\title{
Real-space formulation of the electrostatic potential and total energy of solids
}

\author{
J.E. Pask* and P.A. Sterne \\ Lawrence Livermore National Laboratory, \\ University of California, Livermore, California 94550
}

(Dated: 23 Dec 2004)

\begin{abstract}
We develop expressions for the electrostatic potential and total energy of crystalline solids which are amenable to direct evaluation in real space. Unlike conventional reciprocal space formulations, no Fourier transforms or reciprocal lattice summations are required, and the formulation is well suited for large-scale, parallel computations. The need for reciprocal space expressions is eliminated by replacing long-range potentials by equivalent localized charge distributions and incorporating long-range interactions into boundary conditions on the unit cell. In so doing, a simplification of the conventional reciprocal space formalism is obtained. The equivalence of the real- and reciprocal space formalisms is demonstrated by direct comparison in self-consistent density-functional calculations.
\end{abstract}

PACS numbers: 71.15.-m, 71.15.Nc, 71.15.Ap 
The evaluation of the electrostatic potential and total energy of crystalline solids has been an ongoing problem since the earliest days of solid state physics. ${ }^{1-6}$ In $a b$ initio densityfunctional ${ }^{7,8}$ calculations, the electrostatic component of the potential is typically written as a sum of ionic (or nuclear, in the all-electron context) and electronic (Hartree) terms. In an infinite crystal, however, each of these terms diverges and the sum is only conditionally convergent due the long-range $1 / r$ nature of the Coulomb interaction. Similarly, the electrostatic component of the total energy is a sum of electron-ion, electron-electron, and ion-ion terms, each of which diverges in an infinite crystal but combine to yield a finite total electrostatic energy per unit cell.

It has been appreciated for some time that the divergences and conditional convergence of such extended lattice summations can be eliminated by formulating the summations in terms of neutral densities that are well localized in real and/or reciprocal (Fourier) space. ${ }^{2}$ In the conventional reciprocal space approach for ab initio calculations of crystalline solids, ${ }^{6,9}$ divergences are eliminated by adding neutralizing densities to otherwise divergent Coulomb terms in such a way that the effects of the added densities cancel in the final expressions. Remaining long-range interactions are then rendered short ranged by transforming to reciprocal space, where smooth periodic functions, of infinite extent in real space, are well localized. The resulting expressions for the electrostatic potential and total energy contain structure factors and/or Ewald sums, and require at least $O(N \log N)$ operations to evaluate, where $N$ is the number of atoms in the unit cell. Furthermore, since the reciprocal space approach uses a Fourier basis, boundary conditions are necessarily periodic in all directions. And since the approach relies on Fourier transforms, it is difficult to implement efficiently on large-scale parallel computational architectures due the need for extensive interprocessor communications.

The limitations of the reciprocal space approach have inspired much research on realspace and local-orbital based approaches ${ }^{10-27}$ which allow for better scaling, a variety of boundary conditions, and eliminate the need for Fourier transforms. In order to treat longrange Coulomb interactions in crystals, these approaches also generally formulate otherwise divergent summations and integrals in terms of neutral densities. Alemany et al. ${ }^{11}$ employ a uniform neutralizing density in a real-space formulation of the Hartree term. Ionic terms are, however, computed as in the conventional reciprocal space formalism, using Fourier transforms. Other real-space formulations have employed localized neutralizing densities to eliminate the need for reciprocal space transformations altogether. Kane ${ }^{25}$ employs neutralizing Gaussian densities to construct a rapidly convergent expression for the crystal potential and finite total Coulomb energy in the context of a real-space Wannier function approach. The formulation of Bachelet et al. ${ }^{26}$ employs Gaussian representations of local ionic densities to construct total densities and potentials and associated neutral terms amenable to evaluation in real space. Tsuchida and Tsukada ${ }^{23}$ employ a combination of localized and uniform neutralizing densities to construct neutral terms optimized for evaluation in real space. Ordejón et al. ${ }^{27}$ form neutral terms by expressing potentials and energies in terms of neutral pseudoatomic densities and differences of the crystal density from these. The formulation of Fattebert and Nardelli ${ }^{20}$ employs neutralizing Gaussian densities and associated potentials, in the spirit of the classical Ewald method, to render long-range interactions short ranged.

Here, we develop expressions for the electrostatic potential and total energy of a crystalline solid which are amenable to direct evaluation in real space in $O(N)$ operations. The expression for the total energy so obtained is variational in the output density and quadratically convergent. We eliminate the need for reciprocal space transformations and/or neu- 
tralizing analytic functions by replacing long-range potentials by the localized charge distributions which generate them, and incorporating long-range interactions into boundary conditions on the unit cell. In so doing, we obtain a simplification of the conventional reciprocal space formalism.

In the pseudopotential approximation, ${ }^{9}$ the Kohn-Sham equations of density functional theory $^{7,8}$ are given by

$$
\begin{aligned}
& -\frac{1}{2} \nabla^{2} \psi_{i}(\mathbf{x})+V_{e f f} \psi_{i}(\mathbf{x})=\varepsilon_{i} \psi_{i}(\mathbf{x}) \\
& V_{e f f}=V_{I}^{l}+V_{I}^{n l}+V_{H}+V_{x c} \\
& V_{I}^{l}=\sum_{a} V_{I, a}(\mathbf{x}) \\
& V_{I}^{n l} \psi_{i}=\sum_{a} \int d \mathbf{x}^{\prime} V_{I, a}^{n l}\left(\mathbf{x}, \mathbf{x}^{\prime}\right) \psi_{i}\left(\mathbf{x}^{\prime}\right), \\
& V_{H}=-\int d \mathbf{x}^{\prime} \frac{\rho_{e}\left(\mathbf{x}^{\prime}\right)}{\left|\mathbf{x}-\mathbf{x}^{\prime}\right|} \\
& V_{x c}=V_{x c}\left(\mathbf{x} ; \rho_{e}\right) \\
& \rho_{e}=-\sum_{i} f_{i} \psi_{i}^{*}(\mathbf{x}) \psi_{i}(\mathbf{x})
\end{aligned}
$$

where $\psi_{i}$ and $\varepsilon_{i}$ are the Kohn-Sham eigenfunctions and eigenvalues, $V_{I, a}$ and $V_{I, a}^{n l}$ are the local and nonlocal parts of the ionic pseudopotential of atom $a, \rho_{e}$ is the electronic charge density, the integrals extend over all space, and the summations extend over all atoms $a$, and states $i$ with occupations $f_{i}$. (Atomic units are used throughout.) For simplicity, we omit spin and crystal momentum indices and consider the case in which the external potential arises from the ions. The nonlocal part $V_{I}^{n l}$ and exchange-correlation potential $V_{x c}$ are determined by the choice of pseudopotentials and exchange-correlation functional, respectively. $V_{I}^{l}$ is the Coulomb potential arising from the ions and $V_{H}$ is that arising from the electrons (Hartree potential).

In an infinite crystal, $V_{I}^{l}$ and $V_{H}$ are divergent and the total Coulomb potential $V_{C}=$ $V_{I}^{l}+V_{H}$ within the unit cell depends on ions and electrons far from the unit cell due to the long-range $1 / r$ nature of the Coulomb interaction. The latter constitutes a particular problem for real-space formulations. Both difficulties may be overcome, however, by replacing the long-range ionic potentials by the short ranged charge densities which generate them, and incorporating long-range interactions into boundary conditions on the unit cell. By construction, the local ionic pseudopotentials $V_{I, a}$ of each atom $a$ vary as $-Z_{a} / r$ (or rapidly approach this) outside their respective pseudopotential cutoff radii $r_{c, a}$; where $Z_{a}$ is the effective ionic charge and $r$ is the radial distance. They thus correspond, by Poisson's equation, to charge densities $\rho_{I, a}$ strictly localized within $r_{c, a}$ (or rapidly approaching this). The local ionic potentials, both inside and outside $r_{c, a}$, may thus be replaced by corresponding charge densities localized within $r_{c, a}$. Figure 1 shows a typical local ionic pseudopotential and corresponding ionic charge density. The total ionic charge density is then

$$
\rho_{I}=\sum_{a} \rho_{I, a}(\mathbf{x})
$$

where the summation extends over all atoms in the crystal. Since the ionic densities are localized in real space, however, the summation in the unit cell is in fact finite and readily 


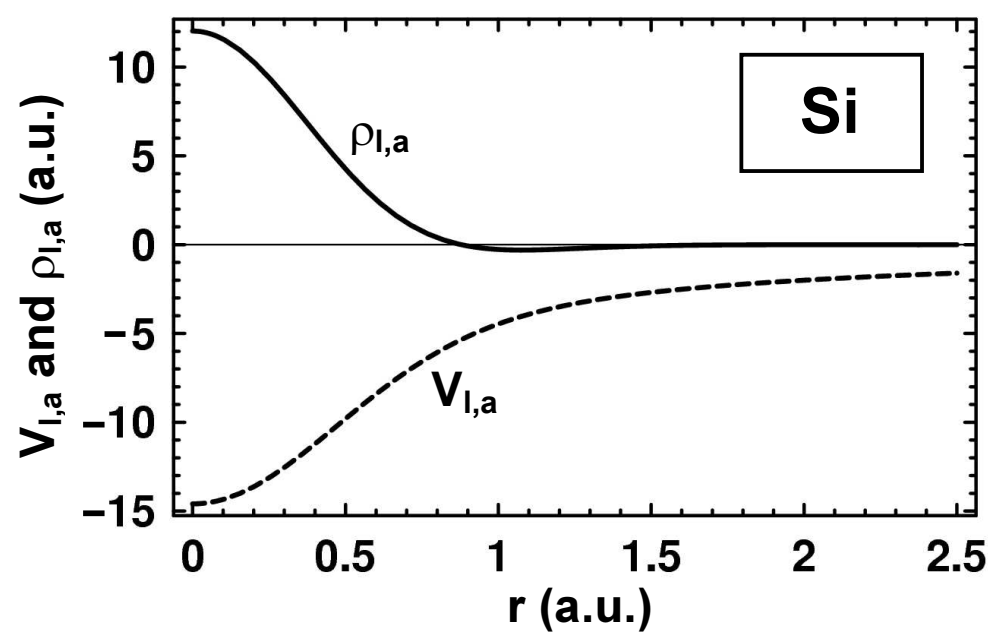

FIG. 1: Local part $V_{I, a}$ of Si pseudopotential ${ }^{29}$ and corresponding localized charge density $\rho_{I, a}$. The potential has a long-range $1 / r$ tail whereas the corresponding density is localized in real space. The total ionic density in the unit cell is thus readily summed in real space whereas the total ionic potential is not.

performed in real space, unlike the summation of ionic potentials. Having constructed the ionic charge density in the unit cell, the total charge density $\rho=\rho_{I}+\rho_{e}$ may then be constructed and the total Coulomb potential $V_{C}=V_{I}^{l}+V_{H}$ may be computed at once by a single Poisson solution subject to periodic boundary conditions:

$$
\nabla^{2} V_{C}(\mathbf{x})=4 \pi \rho(\mathbf{x})
$$

whereupon $V_{\text {eff }}$ may be evaluated as in (2).

The above formulation exploits the fact that, although long-range in real space, the $1 / r$ nature of the ionic potentials outside $r_{c}$ makes them physically equivalent to ionic densities localized within $r_{c}$; and that, although depending physically on all ions and electrons in the crystal, the total Coulomb potential in the unit cell is determined completely by the total charge density within the cell, and the boundary conditions it must satisfy. In this sense, the contributions from all charges outside the cell are folded into the boundary conditions on the cell, as in the reciprocal space component of the classical Ewald formulation ${ }^{2}$ and other more recent reciprocal ${ }^{3}$ and real $^{14}$ space approaches. By computing the total Coulomb potential at once, individual divergences are eliminated. By formulating it in terms of localized functions within the unit cell, its evaluation can be accomplished in $O(N)$ operations, since the ionic charge at each point in the cell is contributed by a fixed number of neighboring atoms and the Poisson solution can be accomplished in $O(N)$ operations in real space by multilevel methods. ${ }^{15}$

In the pseudopotential approximation, the total energy in density-functional theory is given by

$$
\begin{aligned}
& E_{t o t}=T_{s}+E_{e I}^{l}+E_{e I}^{n l}+E_{e e}+E_{I I}+E_{x c} \\
& T_{s}=\sum_{i} f_{i} \int d \mathbf{x} \psi_{i}^{*}(\mathbf{x})\left(-\frac{1}{2} \nabla^{2}\right) \psi_{i}(\mathbf{x}) \\
& E_{e I}^{l}=-\int d \mathbf{x} \rho_{e}(\mathbf{x}) V_{I}^{l}(\mathbf{x})
\end{aligned}
$$




$$
\begin{aligned}
& E_{e I}^{n l}=\sum_{i} f_{i} \int d \mathbf{x} \psi_{i}^{*}(\mathbf{x}) V_{I}^{n l} \psi_{i}(\mathbf{x}), \\
& E_{e e}=\frac{1}{2} \iint d \mathbf{x} d \mathbf{x}^{\prime} \frac{\rho_{e}(\mathbf{x}) \rho_{e}\left(\mathbf{x}^{\prime}\right)}{\left|\mathbf{x}-\mathbf{x}^{\prime}\right|}, \\
& E_{I I}=\frac{1}{2} \sum_{a, a^{\prime} \neq a} \frac{Z_{a} Z_{a^{\prime}}}{\left|\boldsymbol{\tau}_{a}-\boldsymbol{\tau}_{a^{\prime}}\right|} \\
& E_{x c}=-\int d \mathbf{x} \rho_{e}(\mathbf{x}) \varepsilon_{x c}\left(\mathbf{x} ; \rho_{e}\right),
\end{aligned}
$$

where $Z_{a}$ is the ionic charge of atom $a$ at position $\tau_{a}$ and, as in (1)-(7), the integrals extend over all space, and the summations extend over all atoms $a$ and $a^{\prime}$, and states $i$ with occupations $f_{i}$. $T_{s}$ is the kinetic energy of the non-interacting system; $E_{e I}^{l}, E_{e e}$, and $E_{I I}$ are the potential energies associated with the Coulomb interaction between electrons and ions, electrons and electrons, and ions and ions, respectively; $E_{e I}^{n l}$ is the energy associated with the nonlocal part of the ionic potential; and $E_{x c}$ is the exchange-correlation energy. $T_{s}$ is determined by the Kohn-Sham orbitals and occupations, $E_{e I}^{n l}$ is determined by the choice of pseudopotentials, and $E_{x c}$ is determined by the choice of exchange-correlation functional.

In an infinite crystal, the total energy per unit cell may be obtained by restricting the integrals over $\mathbf{x}$ and summation on $a$ in (11)-(16) to the unit cell, while the integrals over $\mathbf{x}^{\prime}$ and summation on $a^{\prime}$ remain over all space. In this case, $E_{e I}^{l}$ is divergent and negative while $E_{e e}$ and $E_{I I}$ are divergent and positive due to the long-range $1 / r$ nature of the Coulomb interaction. However, in terms of the total charge density $\rho$ and Coulomb potential $V_{C}$, the finite total Coulomb energy per unit cell $E_{C}=E_{e I}^{l}+E_{e e}+E_{I I}$ may be obtained at once:

$$
E_{C}=-\frac{1}{2} \int_{\Omega} d \mathbf{x} \rho(\mathbf{x}) V_{C}(\mathbf{x})-E_{s},
$$

where $\Omega$ is the unit cell and $E_{s}$ is the ionic self-energy per unit cell. The ionic self-energy is subtracted so that $E_{C}$ corresponds to the conventional density-functional Coulomb energy, which excludes ionic self-energy. This self energy may be computed from the ionic potentials and associated densities:

$$
E_{s}=-\frac{1}{2} \sum_{a} \int d \mathbf{x} \rho_{I, a}(\mathbf{x}) V_{I, a}(\mathbf{x}),
$$

where the summation is over atoms in the unit cell and the integrals are over all space. The integrals are readily evaluated as one dimensional radial integrals over a finite interval by virtue of the spherical symmetry and short range of the ionic densities. The remaining ion-ion energy in (17) corresponds to the point-ion energy (15) by virtue of the localization of the ionic charge densities within their respective pseudopotential cutoff radii: consistent with the frozen core approximation, there is negligible overlap. ${ }^{33}$

The above formulation exploits the fact that, although the total Coulomb energy per unit cell depends physically on the contributions of ions and electrons throughout the crystal, it is determined completely, per unit cell, by the density and potential within the unit cell. Thus the need for reciprocal space transforms, structure factor computations, and/or Ewald sums is eliminated and the evaluation can be accomplished in $O(N)$ operations in real space.

In terms of the total Coulomb energy, the total energy per unit cell is then

$$
E_{t o t}=T_{s}+E_{C}+E_{e I}^{n l}+E_{x c} .
$$


The orbital dependence in $T_{s}$ and $E_{e I}^{n l}$ can be eliminated in the usual way using the KohnSham equations to obtain the relation

$$
T_{s}-\int_{\Omega} d \mathbf{x} \rho_{e}(\mathbf{x}) V_{e f f}^{l}(\mathbf{x})+E_{e I}^{n l}=\sum_{i} f_{i} \varepsilon_{i},
$$

where $V_{\text {eff }}^{l}$ is the local part of the effective potential which produces Kohn-Sham orbitals $\psi_{i}$ and eigenvalues $\varepsilon_{i}$ according to (1), and $\rho_{e}$ is the electronic charge density corresponding to orbitals $\psi_{i}$ according to $(7)$. Combining (16)-(20), we arrive then at an explicit real-space expression for the total energy per unit cell in terms of the Kohn-Sham eigenvalues:

$$
\begin{aligned}
E_{t o t}=\sum_{i} f_{i} \varepsilon_{i}+\int_{\Omega} d \mathbf{x}\left[\rho_{e}(\mathbf{x}) V_{e f f}^{l}(\mathbf{x})-\frac{1}{2} \rho(\mathbf{x}) V_{C}(\mathbf{x})-\rho_{e}(\mathbf{x}) \varepsilon_{x c}\left(\mathbf{x} ; \rho_{e}\right)\right] \\
+\frac{1}{2} \sum_{a} \int d \mathbf{x} \rho_{I, a}(\mathbf{x}) V_{I, a}(\mathbf{x}) .
\end{aligned}
$$

In the self-consistent solution process, $V_{\text {eff }}^{l}$ is the local part of the effective potential which produces Kohn-Sham orbitals $\psi_{i}$ and eigenvalues $\varepsilon_{i}$. The electronic charge density $\rho_{e}$ is constructed from the orbitals, the total charge density $\rho$ is constructed from $\rho_{e}$, and the total Coulomb potential $V_{C}$, from $\rho$. With such accounting of self-consistent inputs and outputs, the input-density/potential dependence in the first term of (21) is exactly cancelled by the second, and the resulting expression is precisely equal to the Kohn-Sham functional (10) for a given input $V_{\text {eff }}$. It is thus variational in the output density $\rho_{e}$ and quadratically convergent. ${ }^{9,28}$ Furthermore, it can be evaluated in $O(N)$ operations for a given Kohn-Sham spectrum and density.

To verify the equivalence of the present real-space formulation to the conventional reciprocal space formulation, we have implemented it in a finite-element electronic structure code ${ }^{24}$ and compared eigenvalues and total energies to those produced by an established planewave code. $^{30,31}$ Figure 2 shows the convergence of the total energy and lowest few eigenvalues produced by the real-space formulation to those produced by the reciprocal space formulation in a self-consistent crystalline GaAs calculation using the same pseudopotentials ${ }^{29}$ and exchange-correlation functional. ${ }^{32}$ In this case, the reciprocal space results were converged to $10^{-9} \mathrm{Ha}$ and so were taken as "exact" for the purposes of comparison. The real-space basis was then successively refined in a series of self-consistent calculations to allow analysis of the convergence of the real-space results to exact values. As the figure shows, both eigenvalues and total energy converge to the exact reciprocal space values over the full range explored, and do so at the optimal theoretical rate consistent with the cubic completeness of the real-space basis.

In summary, we have derived expressions for the electrostatic potential and total energy of crystalline solids which are amenable to direct evaluation in real space. Unlike conventional reciprocal space formulations, no Fourier transforms or reciprocal lattice sums are required, and the formulation is well suited for large-scale, parallel computations. The need for reciprocal space expressions is eliminated by replacing long-range potentials by equivalent localized charge distributions and incorporating long-range interactions into boundary conditions on the unit cell. In so doing, a simplification of the conventional reciprocal space formalism is obtained and the resulting expressions can be evaluated directly in real space in $O(N)$ operations. 


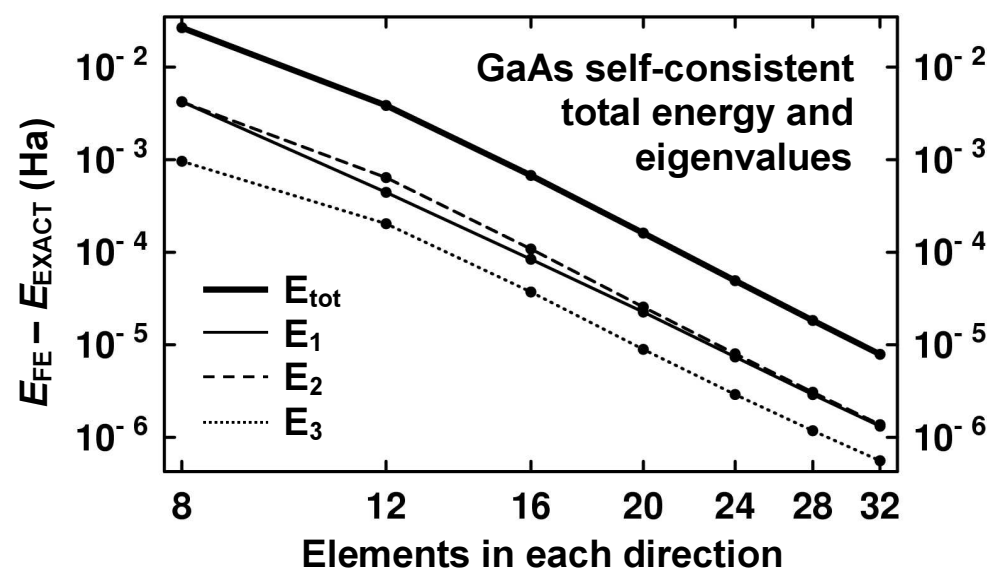

FIG. 2: Convergence of real-space total energy and eigenvalues to exact values in a self-consistent GaAs calculation. Here, "exact values" were obtained from a highly converged planewave calculation; and real-space values, from a series of finite-element calculations. The asymptotic slope of $\sim-6$ on the log-log scale shows that both total energy and eigenvalues converge to exact values at the optimal theoretical rate consistent with the cubic completeness of the finite-element basis: the error is $O\left(h^{6}\right)$, where $h$ is the mesh spacing.

While the development here has been in the context of density-functional theory, and has been demonstrated by finite-element calculations, the ideas and/or expressions so obtained are applicable within a broad range of interaction models and basis sets.

\section{Acknowledgments}

We thank L.H. Yang and W.E. Pickett for helpful comments and discussions. This work was performed under the auspices of the U.S. Department of Energy by University of California, Lawrence Livermore National Laboratory under Contract W-7405-Eng-48.

* Electronic address: pask1@llnl.gov

1 E. Madelung, Phys. Z. 19, 524 (1918).

2 P.P. Ewald, Ann. Phys. 64, 253 (1921); Göttinger Nachr., Math.-Phys. Kl. II 3, 55 (1937).

3 M. Weinert, J. Math. Phys. 22, 2433 (1981).

4 E. Wigner and F. Seitz, Phys. Rev. 43, 804 (1933); 46, 509 (1934).

5 K. Fuchs, Proc. R. Soc. 151, 585 (1935).

6 J. Ihm, A. Zunger, and M.L. Cohen, J. Phys. C 12, 4409 (1979).

7 P. Hohenberg and W. Kohn, Phys. Rev. 136, B864 (1964); W. Kohn and L.J. Sham, Phys Rev. 140, A1133 (1965).

8 R.O. Jones and O. Gunnarsson, Rev. Mod. Phys. 61, 689 (1989).

9 W.E. Pickett, Comput. Phys. Rep. 9, 115 (1989).

10 J.R. Chelikowsky, N. Troullier, and Y. Saad, Phys. Rev. Lett. 72, 1240 (1994); J.R. Chelikowsky, N. Troullier, K. Wu, and Y. Saad, Phys. Rev. B 50, 11355 (1995).

11 M.M.G. Alemany, M. Jain, L. Kronik, and J.R. Chelikowsky, Phys. Rev. B 69, 075101 (2004). 
12 A.P. Seitsonen, M.J. Puska, and R.M. Nieminen, Phys. Rev. B 51, 14057 (1995); M. Heiskanen, T. Torsti, M.J. Puska, and R.M. Nieminen, Phys. Rev. B 63, 245106 (2001).

13 F. Gygi and G. Galli, Phys. Rev. B 52, R2229 (1995).

14 K.A. Iyer, M.P. Merrick, and T.L. Beck, J. Chem. Phys. 103, 227 (1995).

15 T.L. Beck, Rev. Mod. Phys. 72, 1041 (2000).

16 T. Hoshi, M. Arai, and T. Fujiwara, Phys. Rev. B 52, R5459 (1995).

17 E.L. Briggs, D.J. Sullivan, and J. Bernholc, Phys. Rev. B 52, R5471 (1995); 54, 14362 (1996).

18 N.A. Modine, G. Zumbach, and E. Kaxiras, Phys Rev. B 55, 10289 (1997); U.V. Waghmare, H. Kim, I.J. Park, N. Modine, P. Maragakis, and E. Kaxiras, Comput. Phys. Commun. 137, 341 (2001).

19 J.-L Fattebert, J. Comput. Phys. 149, 75 (1999).

20 J.-L. Fattebert and M.B. Nardelli, in Handbook of Numerical Analysis, vol. X, Elsevier, Amsterdam, 2003.

21 F. Ancilotto, P. Blandin, and F. Toigo, Phys. Rev. B 59, 7868 (1999).

22 T. Ono and K. Hirose, Phys. Rev. Lett. 82, 5016 (1999).

23 E. Tsuchida and M. Tsukada, Phys. Rev. B 52, 5573 (1995); J. Phys. Soc. Japan 67, 3844 (1998).

24 J.E. Pask, B.M. Klein, C.Y. Fong, and P.A. Sterne, Phys. Rev. B 59, 12352 (1999); J.E. Pask, B.M. Klein, P.A. Sterne, and C.Y. Fong, Comput. Phys. Commun. 135, 1 (2001).

25 E.O. Kane, Phys. Rev. B 21, 4600 (1980).

26 G.B. Bachelet, H.S. Greenside, G.A. Baraff, and M. Schlüter, Phys. Rev. B 24, 4745 (1981); G.A. Baraff and M. Schlüter, Phys. Rev. B 28, 2296 (1983); G.A. Baraff and M. Schlüter, Phys. Rev. B 30, 1853 (1984).

27 P. Ordejón, E. Artacho, and J.M. Soler, Phys. Rev. B 53, 10441 (1996); J.M. Soler, E. Artacho, J.D. Gale, A. García, J. Junquera, P. Ordejón, D. Sánchez-Portal, J. Phys.: Condens. Matter 14, 2745 (2002).

28 J.R. Chelikowsky and S.G. Louie, Phys. Rev. B 29, 3470 (1984).

29 C. Hartwigsen, S. Goedecker, and J. Hutter, Phys. Rev. B 58, 3641 (1998).

30 X. Gonze, J.-M. Beuken, R. Caracas, F. Detraux, M. Fuchs, G.-M. Rignanese, L. Sindic, M. Verstraete, G. Zerah, F. Jollet, M. Torrent, A. Roy, M. Mikami, Ph. Ghosez, J.-Y. Raty, and D.C. Allan, Comput. Mat. Sci. 25, 478 (2002).

31 The ABINIT code is a common project of the Université Catholique de Louvain, Corning Incorporated, and other contributors (URL http://www.abinit.org).

32 J.P. Perdew and A. Zunger, Phys. Rev. B 23, (1981).

33 If it were desired to employ pseudopotentials with non-negligible core overlap, a correction is readily added in terms of 2-center integrals (see, e.g., O.F. Sankey and D.J. Niklewski, Phys. Rev. B 40, 3979 (1989) in the context of pseudoatomic orbitals). 